\title{
QUADERNI Quaderni
}

Communication, technologies, pouvoir

101 | Été 2020

La fin des communicants politiques?

\section{Un renouveau des conseillers en com'?}

\section{Camila Moreira Cesar et Pierre-Emmanuel Guigo}

\section{(2) OpenEdition}

\section{Journals}

Édition électronique

URL : https://journals.openedition.org/quaderni/1657

DOI : 10.4000/quaderni.1657

ISSN : 2105-2956

\section{Éditeur}

Les éditions de la Maison des sciences de l'Homme

\section{Édition imprimée}

Date de publication : 5 octobre 2020

Pagination : 9-15

\section{Référence électronique}

Camila Moreira Cesar et Pierre-Emmanuel Guigo, « Un renouveau des conseillers en com' ? », Quaderni [En ligne], 101 | Été 2020, mis en ligne le 05 octobre 2020, consulté le 05 janvier 2023. URL : http:// journals.openedition.org/quaderni/1657 ; DOI : https://doi.org/10.4000/quaderni.1657 


\section{$D$ ossier}

\section{Un renouveau des conseillers en com'?}

Depuis une dizaine d'années on ne compte plus les reportages, films, séries télévisées s'intéressant aux conseillers en communication ou spin doctors, pour reprendre l'appellation qui les a rendus célèbres. Dans ces récits mêlant pour la plupart fiction et inspiration d'événements réels, ces acteurs sont présentés comme des spécialistes de la communication exerçant une influence décisive sur les politiques et manipulant à leur aise les journalistes.

En prenant du recul à l'égard de ces représentations médiatiques pour voir ce qu'il en est dans la réalité de la vie politique, ce dossier vise à

\section{Camila Moreira Cesar}

\author{
Université Sorbonne \\ Nouvelle Paris 3
}

\section{Pierre-Emmanuel Guigo}

Université Paris-Est Créteil Centre de recherche en Histoire Européenne Comparée rouvrir une thématique de recherche vivace dans les années 1980-1990. On peut penser notamment aux recherches de Larry Sabato ${ }^{1}$ aux États-Unis et, en France, à la thèse pionnière de Jean-Baptiste Legavre $^{2}$ et aux écrits de Didier Georgakakis ${ }^{3}$. Le thème semblait s'être un peu tari par la suite, à l'exception peut-être de la perspective historique $^{4}$. Pourtant, la dernière décennie a été riche en mutations pour la sphère de la communication politique. Ainsi, une question se pose : peut-on regarder les conseillers en communication politique avec les mêmes lentilles qu'autrefois?

Le développement d'Internet a largement modifié les manières de faire campagne, les formes des mobilisations et les modes de gouvernement ${ }^{5}$. Les technologies de l'information et de la communication ainsi que les médias socionumériques sont devenus des atouts incontournables de la vie politique, obligeant les représentants à s'adapter à un format de communication bien différent de la logique des médias de masse 6 . Cela a notamment conduit à une remise en cause du statut des journalistes comme intermédiaires obligés 
entre la parole politique et les citoyens ${ }^{7}$. Cette nouvelle "écologie communicationnelle ${ }^{8}$ " bouscule de même la place et le rôle de conseillers en communication, désormais confrontés à des dynamiques de communication inédites et à l'imprévisibilité d'une majorité silencieuse devenue « agissante » sur les réseaux.

Si, comme l'affirme Françoise Piotet, le nom de métier "sert à évoquer, de manière la plus synthétique possible, l'occupation et la position sociale ${ }^{9} »$ d'un groupe d'individus dans l'espace social, le changement de position, explique l'auteure, est dès lors accompagné d'un changement de nom, les différentes appellations traduisant un renouvellement des tâches ou des fonctions dans le mouvement de recomposition du travail. S'agissant des spécialistes de la communication, leur histoire montre que, des « conseillers de communication » aux spin doctors en passant par les « attachés de presse » et les experts en « relations publiques», les profils ont changé, aussi bien que les attributions, les objectifs et les conceptions de la communication qui les orientent $t^{10}$. Pour les transfuges du journalisme, autrefois nombreux à ce poste (Thierry Pfister, Jean-Luc Mano, Claude Sérillon, Hélène Fontanaud, Myriam Lévy, Françoise Degois, Bruno Roger-Petit, Pierre Salinger, ou Roger Ailes aux États-Unis), il est plus difficile de justifier un rôle qui s'appuyait largement sur leur carnet d'adresses et la connaissance d'un milieu aujourd'hui moins essentiel que par le passé. Quant aux publicitaires, devenus dans les années 1980 le modèle par excellence des conseillers, leur légitimité fondée sur leur expertise de la télévision - notamment des spots publicitaires et de l'affichage est aussi remise en cause par les nouveaux outils numériques.

Ce « quatrième âge de la communication politique $^{I l} »$, caractérisé par un éclatement de l'information, une accélération du tempo politique, l'accroissement du sentiment anti-élitaire voit-il donc la remise en cause, la disparition des conseillers en communication, héros - hérauts ?de l'âge audiovisuel ? Ou, au contraire, la nature fluctuante des rôles assumés par ces acteurs favorise une réinvention des positions qu'ils peuvent occuper dans le fonctionnement de la politique de nos jours? Si tel est le cas, il reste à interroger les formes qu'elles peuvent prendre ainsi que les enjeux auxquels se heurtent ces acteurs dans un environnement social, politique et technologique de plus en plus complexe. Alors que Bernard Manin expliquait au milieu des années 1990 que «la démocratie du public est le règne de l'expert de communication ${ }^{12} »$, les dernières décennies invitent à repenser la place des « promoteurs de la politique ${ }^{13}$ » à l'ère de ce que Philippe Aldrin et Nicolas Hubé dénomment la démocratie des publics : une nouvelle forme du régime représentatif caractérisée par « la diversification des lieux, des modes et des supports d'expression d'une opinion publique qui se donne de moins en moins à voir au singulier ${ }^{14}$.»

Pour mener cette enquête, nous avons aussi voulu décloisonner l'approche. Si la recherche française fut abondante, elle s'intéresse peu aux exemples étrangers et les recherches internationales laissent d'ailleurs souvent de côté le cas français, alors qu'ils font bien sûr la part belle aux États-Unis, à la Grande-Bretagne ou à l'Allemagne. Ce dossier se veut donc résolument international. Le cas français est bien entendu présent, mais 
la majorité des contributions éclaire au moins un autre contexte national. En effet, dans un domaine particulièrement internationalisé, où les équipes de campagne s'observent très largement pour chercher la nouveauté à l'étranger, il nous semblait nécessaire de rendre compte de la diversité de cette communication politique et de ses acteurs tout en soulignant les éventuels transferts internationaux. Le grand modèle pour beaucoup de communicants demeure sans conteste les États-Unis, comme le montre bien le cas britannique (cf. article de Karine Rivière de Franco), mais d'autres pays peuvent aussi être des pôles de rayonnement et inspirer des tendances, comme la France (cf. article d'Ivan Schuliaquer). Surtout, ce dossier met également en valeur un espace jusque-là parent pauvre des études sur la communication politique internationale mais pas moins intéressant : l'Amérique latine. En effet, si Daniel C. Hallin et Paolo Mancini ont tempéré leurs trois modèles de systèmes médiatiques - modèle libéral, modèle nord-européen, modèle méditerranéen - pour l'ouvrir à l'international, les pays d'Amérique latine en étaient absents, à l'exception du Brési $1^{15}$. Le présent numéro offre ainsi un regard au plus près de cette partie de l'Amérique, en explorant les terrains du Brésil (article de Camila Moreira Cesar et Nina Fernandes dos Santos) aussi bien que de l'Uruguay (article d'Ivan Schuliaquer). L'Allemagne était également l'autre grande absente des recherches françaises, ce que les travaux de Nicolas Hubé depuis plusieurs années permettent de compenser, à l'instar de sa contribution dans ce dossier. L'Italie, dont les bouleversements contemporains semblent préfigurer les mutations de nos démocraties libérales occidentales, est plus étudiée en France. Mais la communication du Mouvement
5 étoiles restait pour le moment moins décrite, vide que vient combler l'article de Raffaello Doro.

Cette ouverture internationale, bien que faite de cas et de temporalités politiques différents, permet de faire ressortir quelques évolutions saillantes de cette figure du communicant politique aujourd'hui. Tous les exemples étudiés conduisent à penser que l'émergence d'un champ professionnel du conseil en communication politique reste très lointaine. La diversité des profils et des fonctions demeure en effet une caractéristique marquante de ce milieu d'acteurs. Beaucoup plus que des professionnels de la communication, ce sont des profils plus politisés et plus militants qui semblent s'imposer (cf. article de PierreEmmanuel Guigo), pouvant faire valoir d'autres ressources (proximité, cohérence idéologique, connaissance des campagnes) que celles d'un expert en communication «pur », souvent sujet à défiance ${ }^{16}$. La professionnalisation d'une activité de political consultant ou campaign manager semble donc spécifique aux États-Unis, même si d'autres pays semblent l'imiter (cf. article de Karine Rivière de Franco).

Ce numéro permet également de revaloriser le rôle du politique dans l'élaboration de cette communication politique. L'image caricaturale du conseiller tirant les ficelles de l'opinion, mais aussi des élus et dirigeants laissait penser à une perte de pouvoir du politique sur l'expert en communication. Il ressort de nos différents articles que le politique est loin d'être un pantin, même si la présence des communicants dans les enceintes du pouvoir se présente comme une nécessité face aux évolutions de la médiatisation 
du politique. Cependant, comme le dévoile la contribution de Ivan Schuliaquer à propos du cas du Frente Amplio en Uruguay, la gestion de la communication peut aussi se faire de façon plus « artisanale », en dehors de tout recours à des conseillers en communication, montrant qu'il n'y a donc pas de fatalité à l'institutionnalisation du rôle de ces « montreurs de communication ${ }^{17}$.»

Très différents les uns des autres, les six cas étudiés permettent également de voir des degrés différents de « modernisation» des campagnes dont les caractéristiques tendent à se répandre dans les cycles longs de la vie politique. Dans son ouvrage de référence The virtuous circle $^{18}$, Pippa Norris distinguait ainsi trois types historiques de campagnes électorales : les campagnes pré-modernes basées sur une faible préparation et un budget modeste, privilégiant les événements locaux et les moyens de communication traditionnels; les campagnes modernes reposant sur une organisation nationale, l'importance des sondages et de la télévision, et une professionnalisation des équipes de campagne; et enfin les campagnes postmodernes se caractérisant par des campagnes permanentes, le recours constant aux mesures d'opinion, des budgets conséquents et une professionnalisation interne, mais aussi par des consultants extérieurs.

La dernière catégorie, appelée à se généraliser pour l'auteure, semble essentiellement propre aux États-Unis, et éventuellement à la GrandeBretagne, le pays le plus " professionnalisé » et où le rôle de conseiller en communication - en l'occurrence les SpAds, étudiés par Karine Rivière de Franco dans ce numéro - semble le plus proche du modèle des États-Unis. Le
Mouvement 5 étoiles décrit par Raffaello Doro présente une communication très professionnalisée, mais dans un secteur bien précis, celui du numérique. Les cas allemands, français et italiens se rapprochent plutôt du modèle des campagnes « modernes », tout en incluant certains éléments des campagnes postmodernes (importance prise par les médias numériques, les sondages), mais relevant plutôt du pré-moderne pour d'autres, à l'instar du rôle de la presse en Allemagne, comme le démontre l'article de Nicolas Hubé. Quant au cas uruguayen, étudié par Ivan Schuliaquer à partir de la communication de Tabaré Vázquez et de José « Pepe » Mujica, il semble plutôt proche des campagnes pré-modernes, où le candidat gère directement ses relations avec la presse, sans véritable professionnalisation de l'équipe de campagne.

L'émergence des réseaux socionumériques bouleverse incontestablement les conseillers en communication. Ces experts de la publicité ou des relations presse doivent s'adapter à un monde nouveau. Le rapport aux journalistes, qui était le gros de leur travail autrefois, semble moins crucial (cf. article de Raffaello Doro), ce qui conduit à une plus faible présence des anciens journalistes à ce rôle (cf. article de Pierre-Emmanuel Guigo). Les outils numériques semblent être devenus le nouvel enjeu essentiel des campagnes récentes, comme le démontrent Camila Moreira Cesar et Nina Fernandes dos Santos dans leur étude sur l'usage de l'application WhatsApp comme dispositif de communication politique dans les dernières élections brésiliennes, et Raffaello Doro qui examine, quant à lui, le rôle du web dans l'apparition et l'ancrage du Movimento 5 Stelle dans le paysage politique italien. La 
relation plus directe entre personnel politique et citoyens que valorise ce type de support met encore plus à distance le conseiller en communication " classique », dont le rôle d'interprète de l'opinion devient moins important. On voit toutefois émerger de nouveaux profils et tâches assurées par des conseillers en communication spécialisés dans le domaine numérique. Ils sont souvent plus jeunes et nés avec l'informatique. Souvent présentés comme techniciens, ils n'en sont pas moins socialisés politiquement et leur recrutement se fait d'ailleurs souvent en interne, dans les cercles d'interconnaissance politiques, comme l'aborde Pierre-Emmanuel Guigo dans sa contribution.

Quant à la circulation des fausses informations à l'ère numérique, elle semble contrarier le rôle des spécialistes de la communication. Dans la plupart des cas étudiés, ces acteurs semblent dépassés par ce nouvel enjeu et le subissent plus qu'ils ne le maîtrisent. Ce phénomène a trouvé dans les plateformes numériques un espace propice à leur propagation à une échelle et vitesse inédites, devenant par là même un outil efficace pour l'orchestration des campagnes négatives voire de désinformation. La création des cellules de veille et de riposte tant dans les équipes de campagne que les cabinets politiques pilotés par des communicants témoigne de la nature polyvalente de leur rôle (cf. article de Pierre-Emmanuel Guigo). Pourtant, leurs connaissances et méthodes semblent limitées vis-à-vis des dynamiques d'une communication de plus en plus automatisée.

Cette recherche n'est toutefois qu'un premier pas. Elle appelle d'autres développements, pour confirmer ou infirmer les hypothèses que nous avons voulu présenter ici. D'autres sphères géographiques restent encore peu explorées, en tout cas dans la recherche francophone, comme l'espace asiatique, l'Afrique ou le monde arabe. La comparaison étant l'un des impératifs pour les recherches autour de la communication politique, des études ouvertes à d'autres réalités nationales et régionales permettraient d'éclairer les différences et similitudes ainsi que les constances et stagnations à l'égard des évolutions des manières de communiquer en politique de nos jours.

La perspective historique nous paraît aussi une piste à explorer. La comparaison avec d'autres périodes passées, notamment la fin du XIX ${ }^{\mathrm{e}}$ siècle et le début du $X X^{\mathrm{e}}$ siècle, semble laisser voir des points communs intéressants sur l'émergence d'une professionnalisation d'un secteur appelé « propagande ». Si les années 1970-1980 ont été la période des conseillers en communication, puis la décennie suivante des spin doctors, l'entre-deux-guerres n'a-t-elle pas été l'époque des propagandistes ? Plutôt qu'une professionnalisation linéaire du métier de conseiller en communication, ne faudrait-il pas envisager le recours à ces experts en la matière comme un moment d'adaptation à de nouveaux modes de communiquer, avant que la professionnalisation ne s'intègre en interne, dans les partis ou mouvements politiques, ou dans l'intériorisation des nouveaux codes médiatiques par les professionnels de la politique ? La sous-traitance de la communication serait donc plus l'exception politique que la règle.

Enfin, si les recherches s'intéressent au premier chef à l'inventivité des campagnes électorales, la gestion de la communication en situation d'exer- 
cice du pouvoir reste moins étudiée. Les textes d'Ivan Schuliaquer, de Karine Rivière de Franco, de Raffaello Doro et de Nicolas Hubé donnent toutefois des éclairages sur la communication des gouvernants dans des contextes forts distincts. Mais de plus amples études sur les conseillers en communication du pouvoir permettraient de voir si ce rôle s'institutionnalise ou s'il est sujet à des problématiques similaires que lors des campagnes électorales.

Les contributions rassemblées dans ce numéro de Quaderni fournissent ainsi un panorama des questions-clés de la communication politique contemporaine et invitent à mettre en exergue la place, le rôle et le travail des conseillers en communication dans une période où le tempo de la politique s'inscrit dans l'immédiat.

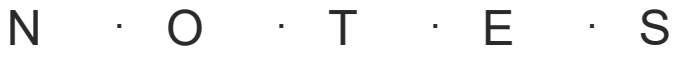

1. Larry J. Sabato, The Rise of Political Consultants, New York, Basic Books, 1981.

2. Jean-Baptiste Legavre, Conseiller en communication politique. L'institutionnalisation d'un rôle, Thèse de doctorat en science politique, Paris, Université Paris I Panthéon-Sorbonne, 1993.

3. Didier Georgakakis, «La double figure des conseils en communication politique. Mises en scène des communicateurs et transformations du champ politique », Sociétés contemporaines, $\mathrm{n}^{\circ 24}$, 1995, p. 77-94.

4. Jérôme Pozzi (dir.), De l'attaché de presse au conseiller en communication. Pour une histoire des spin doctors, Rennes, Presses Universitaires de Rennes, 2019.

5. Fabienne Greffet (dir), Continuerlalutte.com. Les partis politiques sur le Web, Paris, Presses de Sciences Po, 2011 ; Fabienne Greffet, Stéphanie Wojcik, et Gersende Blanchard, «S'engager dans la campagne présidentielle. Les formes multiples de la participation politique en ligne ", Politiques de communication, vol. 3, no. 2, 2014, p. 25-58 ; Anaïs Théviot, « Les data: nouveau trésor des partis politiques? Croyances, constitutions et usages comparés des données numériques au Parti Socialiste et à l'Union pour un Mouvement Populaire », Politiques de communication, vol. 6, $n^{\circ} 1,2016$, p. 137-166 ; Sharon Dawes. « Governance in the digital age: A research and action framework for an uncertain future ", Government Information Quarterly, 26(2), 2009, p. 257-264.

6. Stephen Coleman, « New mediation and direct representation: reconceptualizing representation in the digital age ", New media \& society, vol. 7, n², 2005, p. 177-198; Scott Wright, « From "third place" to "third space": Everyday political talk in non-political online spaces », Javnost - The Public, 2012, vol. 19, 
n³, p. 5-20.

7. Arnaud Mercier, « La lecture événementielle des faits politiques : entre logiques journalistiques et (dés) intermédiation numérique », Sciences de la société, 102, 2017, p. 32-51.

8. Jay G. Blumler, « The Crisis of Public Communication, 1995-2017», Javnost - The Public, vol. 25, $\mathrm{n}^{\circ} 1-2,2018$, p. 1-10.

9. Françoise Piotet, La révolution des métiers, Paris, Presses Universitaires de France, 2002, p. 3.

10. Jérôme Pozzi, «Introduction », in J. Pozzi (dir.), De l'attaché de presse au conseiller en communication, op. cit., p. 7-17.

11. Jay G. Blumler, « The Fourth Age of Political Communication ", Politiques de communication, vol. 6, no. 1, 2016, p. 19-30.

12. Bernard Manin, Principes du gouvernement représentatif, Paris, Flammarion, 1996.

13. Jean-Baptiste Legavre, Conseiller en communication politique..., op.cit.

14. Philippe Aldrin et Nicolas Hubé, Introduction à la communication politique, Louvain-la-Neuve, De Boeck supérieur, coll. Ouvertures politiques, 2017, p. 255.

15. Daniel C. Hallin et Paolo Mancini, Comparing Media system beyond the western world, Cambridge University Press, 2012.

16. Tristan Riffault, « Le consultant et le politique. Le conseil politique en pratique : une approche ethnographique », Sociologie, 2017/3 (Vol. 8), p. 283-300. 17. Érik Neveu, Une société de communication?, Issy-les-Moulineaux, Montchrestien Lextenso éditions, 2011 [1994].

18. Pippa Norris, A Virtuous Circle: Political Communications in Postindustrial Societies, Cambridge, Cambridge University Press, 2000. doi:10.1017/ CBO9780511609343.008. 
